\title{
一种新型科罗索酸衍生物的生物催化合成
}

\author{
付少涁*,a 孟庆峰 ${ }^{b}$ 辛 庆 ${ }^{a}$ 肖世基 ${ }^{a}$ \\ ( ${ }^{a}$ 遵义医学院药学院 遵义 563000) \\ $\left({ }^{b}\right.$ 遵义医学院公共卫生学院 遵义 563000)
}

\begin{abstract}
摘要 科罗索酸是一种五环三萜酸, 因其显著的抗糖尿病活性而备受关注. 通过生物催化的方法对其进行结构修饰和 改造, 增加科罗索酸结构多样性, 为篮选结构新颖或活性更强衍生物奠定基础. 使用一株植物内生真菌 Umbelopsis isabellina 催化合成一个新的科罗索酸衍生物, 命名为 $2 \alpha, 3 \beta, 7 \beta$-三差基-乌苏-11-烯-28,13-内酯(2). 其结构通过高分辨质 谱、核磁共振等波谱技术确定.
\end{abstract}

关键词 科罗索酸; 生物催化; 羟基化; $2 \alpha, 3 \beta, 7 \beta$-三差基-乌苏-11-烯-28,13-内酯

\section{Biocatalytic Synthesis of a Novel Corosolic Acid Derivative}

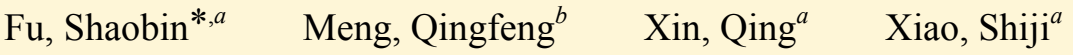 \\ ( ${ }^{a}$ School of Pharmacy, Zunyi Medical University, Zunyi 563000) \\ ( ${ }^{b}$ School of Public Health, Zunyi Medical University, Zunyi 563000)
}

\begin{abstract}
Corosolic acid, a pentacyclic triterpene acid, attracted more and more attention because of the significant anti-diabetes activity. Biocatalysis was applied to modify the structure of corosolic acid to increase the structural diversity of corosolic acid and lay the foundation for screening derivatives with novel structure or better activity. A novel corosolic acid derivative was synthesized by endophytic fungus Umbelopsis isabellina. The structure of the new compound was established by HR-ESIMS and NMR spectrum.
\end{abstract}

Keywords corosolic acid; biocatalysis; hydroxylation; $2 \alpha, 3 \beta, 7 \beta$-trihydroxy-urs-11-en-28,13-lactone

科罗索酸(corosolic acid, CA)又名 $2 \alpha$-羟基熊果酸, 是存在于大叶紫薇、枇杷、对蓦猕猴桃中的一种三萜化 合物. 它以游离形式或㿝式形式存在, 且常与其同分异 构体山楂酸共同存在 ${ }^{[1,2]}$. 由于与其同分异构体的结构 和化学性质相似, 所以给分离、纯化科罗索酸及其衍生 物带来很大困难. 研究发现, 科罗索酸具有抗炎、降血 糖、降血脂和抗癌等作用 ${ }^{[3 \sim 5]}$, 科罗索酸的抗肿瘤作用 已经有很多报道，比如脑瘤、乳腺癌、白血病、肝癌、 肺癌等 $[6,7]$. 但是, 人们最为关注的是科罗索酸的降血糖 功能. 体内、外实验结果表明, 科罗索酸通过增加葡萄 糖的转运, 促进细胞对葡萄糖的吸收和利用, 从而实现 降血糖功效. 科罗索酸对葡萄糖转运的增加作用类似于 胰岛素, 因此, 科罗索酸也被称为 “植物胰岛素” (phyto-insulin, botanical insulin) $^{[8,9]}$.
目前，该天然产物已在美国作为营养补充剂上市， 同时进行治疗糖尿病的临床试验, 科罗索酸作为纯天然 植物活性成分, 既可以作为降血糖保健品, 也可作为降 血糖药物对抗糖尿病和肥胖症进行治疗. 所以科罗索酸 具有很好的开发利用前景, 而对科罗索酸进行结构改造 或者制备其衍生物也成了现在的一个研究热点. 因为科 罗索酸的结构是刚性环, 通过化学方法对其进行结构改 造位点主要集中在 3 位羟基、 28 位羒基等部位. 近年来, 被誉为 “绿色化学” 的生物催化因为其反应条件温和、 副产物少、环境友好等优点越来越多地用于结构修饰和 改造结构复杂的天然产物 ${ }^{[10-12]}$. Feng 等 ${ }^{[1]}$ 用放线菌 Streptomyces asparaginoviolaceus 和丝状 真 菌 Cochliobolus lunatus 转化科罗索酸, 得到 7、21、30 位 等羟基化产物, 且均为新化合物. $\mathrm{Li}$ 等 ${ }^{[12]}$ 通过丝状真菌

* E-mail: fushb@126.com

Received May 9, 2016; revised June 4, 2016; published online July 13, 2016.

Project supported by the National Natural Sciences Foundation of China (No. 21462057), the Program of Ministry of Education "Chunhui Plan" (No. Z2014091), and the United Fund of Guizhou Province, Zunyi Medical University and Zunyi City (No. QKHLH-2014-7555).

国家自然科学基金(No. 21462057)、教育部春晖计划(No. Z2014091)、贵州省科技厅、遵义医学院、遵义市科技局联合基金(No. QKHLH-2014-7555) 资助项目. 
Fusarium equiseti CGMCC 3.3658 和 Gliocladium catenulatum CGMCC 3.3655 得到 4 种结构新颖的科罗索酸衍 生物, 有 15 位的羟基化产物, 有 3 位羟基丢失的产物, 有 3 位 $\beta$ 构型转化成 $\alpha$ 构型产物以及 $\mathrm{A}$ 环扩环产物. 微 生物催化很大程度上增加了科罗索酸的结构多样性. 本 文通过植物内生真菌 Umbelopsis isabellina 与科罗索酸 共培养, 得到一个结构新颖的转化产物(Eq. 1), 经过 HR-ESIMS, 1D 和 2D NMR 等数据鉴定新化合物的结构 为 $2 \alpha, 3 \beta, 7 \beta$-trihydroxy-urs-11-en-28,13-lactone.
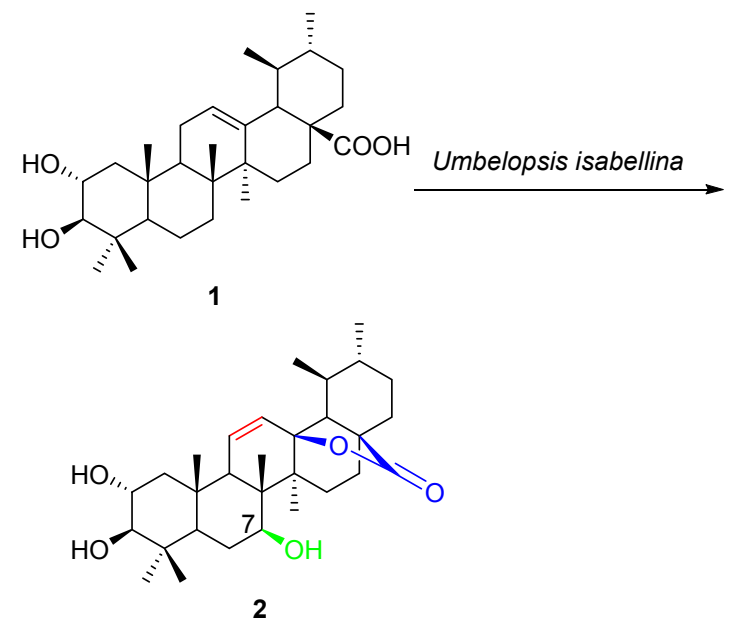

\section{1 结果与讨论}

\section{1 转化反应的篮选}

在篮选的 10 株微生物菌株中, 10 株细菌均未发现 有转化活性, 而 10 株真菌中, 有 2 株真菌经 TLC 方法 [展开剂, $V$ (石油醚) $: V$ (乙酸乙酯 $)=1: 1$, 硫酸乙醇显 色]检测具有转化科罗索酸的活性. 选择其中一种 02-L-37 Umbelopsis isabellina 进行复笁, TLC 结果也显 示其对科罗索酸有转化作用, 故对其进行放大培养并对 转化产物进行结构鉴定.

\section{2 转化产物的结构鉴定}

化合物 1 为白色粉末, 硫酸乙醇 $105{ }^{\circ} \mathrm{C}$ 显色为紫红 色. 高分辨质谱数据(HR-ESI-MS) 测量值 $[\mathrm{M}+\mathrm{H}]^{+}$: 487.3403, 计算值为 487.3423 , 可以推断出转化产物的 分子式为 $\mathrm{C}_{30} \mathrm{H}_{46} \mathrm{O}_{5}$, 提示有 8 个不饱和度. 与底物科罗 索酸 $\left(\mathrm{C}_{30} \mathrm{H}_{48} \mathrm{O}_{4}, 472\right)$ 相比, 转化产物多了一个氧原子, 少了 2 个氢原子. 通过 ${ }^{1} \mathrm{H}$ NMR 发现高场区有 7 个甲基 信号, 底物中 12 位的烯氢质子信号 $\delta 5.49$ (t-like) 消失, 出现两个新的烯氢质子信号 $\delta 6.02(\mathrm{br} \mathrm{d}, J=10.4 \mathrm{~Hz}$, $1 \mathrm{H})$ 和 $\delta 5.71(\mathrm{dd}, J=1.2,9.6 \mathrm{~Hz}, 1 \mathrm{H})$, 以及 3 个连氧碳的 质子信号 $\delta 2.88(\mathrm{~d}, J=9.6 \mathrm{~Hz}, 1 \mathrm{H}), 3.83(\mathrm{dd}, J=4.8,9.6$ $\mathrm{Hz}, 1 \mathrm{H}), 3.63(\mathrm{td}, J=4.8,9.6 \mathrm{~Hz})$, 提示我们转化产物中 有一个位点引入了一个新的羟基. 在 ${ }^{13} \mathrm{C} \mathrm{NMR}$ 谱中, 相
应的出现一对烯碳信号 $\delta 133.6$ 和 130.6, 与底物 CA 中 12 位双键碳的化学位移也不一样 $(\delta 126.0,139.7)$, 有 3 个连氧碳信号 $\delta 84.1,73.6,69.1$, 与 ${ }^{1} \mathrm{H} \mathrm{NMR}$ 一致, 提示 我们有一个新的羟基化位点, 而且双键发生变化. ${ }^{1} \mathrm{H}$ $\mathrm{NMR}$ 和 ${ }^{13} \mathrm{C}$ NMR 显示转化产物与底物比较, 引入了一 个新的羟基, 同时 12 位双键转移, 因为 ${ }^{13} \mathrm{C} N M R$ 出现 一个季碳信号 $\delta 91.9$, 我们推测双键从 12-13 位移至 11-12 位, 同时 13 位发生着基化. 通过 HMQC 谱可知, $\delta_{\mathrm{H}} 2.88(\mathrm{~d}, J=9.6 \mathrm{~Hz})$ 与 $\delta_{\mathrm{C}} 84.1$ 直接相关, $\delta_{\mathrm{H}} 3.63(\mathrm{td}$, $J=4.8,9.6 \mathrm{~Hz})$ 与 $\delta_{\mathrm{C}} 69.3$ 直接相关, 分别与底物中 C(3) 和 C(2)的质子信号和碳信号化学位移一致. HMBC 相关 谱中 $\delta_{\mathrm{C}} 48.3$ 和 38.4 分别为 $\mathrm{C}(14)$ 和 $\mathrm{C}(10)$ 的化学位移. $\delta_{\mathrm{H}}$ 6.02 与 $\delta_{\mathrm{C}} 91.9,48.3,38.4$ 有远程相关, 推测 $\delta_{\mathrm{H}} 6.02$ 为 12 位质子信号, 与我们预期双键移至 11-12 位, C-13 相 应的羟基化的结构相符. 引入羟基我们通过 26 位甲基 $\delta_{\mathrm{H}} 3.83$ 与 $\delta_{\mathrm{C}} 73.6$ 的远程相关推断羟基位于 7 位碳原子 上, 7 位上的羟基定为 $\beta$ 构型是由 H-7 的峰形及偶合常数 确定. H-7 如果为 $\alpha$ 构型, 其偶合常数为一大一小偶合, 与实测值 4.8、9.6 Hz 一致, H-7 如果为 $\beta$ 构型, 那么 $\mathrm{H}-7$ 通常表现为宽单峰或偶合常数相近的 $\mathrm{dd}$ 峰. 此外 7 位差 基的 $\beta$ 构型与很多文献一致 ${ }^{[11,13 \sim 15]}$. 双键移位至 $\mathrm{C}(11)$ $\mathrm{C}(12)$ 位后 13 位羟基可以有两种方式: 一种是 13 位羟基 游离存在, 另一种是 13 位羟基与 28 位羧基脱水形成内 酯. 如果 13 位羟基游离, 分子量应该为 488 , 如果 13 位 差基形成内酯则分子量应该为 486, 而我们实测的质谱 $487.3403[\mathrm{M}+\mathrm{H}]^{+}$, 符合后一种情况, 即 13 位羟基与 28 位羧基脱水形成内酯. 此外, 相同菌株催化相似五环三 萜熊果酸也发生类似的反应 ${ }^{[13]}$. 通过转化产物的 NMR、 HR-ESI-MS 等数据以及化合物 $3 \beta, 7 \beta$-dihydroxy-urs-11en-28,13-lactone 图谱, 最终确定转化产物为 $2 \alpha, 3 \beta, 7 \beta$-三 羟基-乌苏-11-烯-28,13-内酯(图 2), 是一个新的化合物.

\section{2 结论}

内生真菌 02-L-37 Umbelopsis isabellina 对 CA 有转 化作用, $2 \alpha, 3 \beta, 7 \beta$-三羟基-乌苏- 11 -烯- 28,13 -内酯为一个 新化合物. 通过微生物转化可以增加科罗索酸结构多样 性.

\section{3 实验部分}

\section{1 仪器与试剂}

高分辨质谱用 Bruker 型质谱仪测定; 核磁共振用 Agilent DD2400-MR 型核磁共振仪测定, TMS 为内标; 薄层色谱硅胶 GF254 和柱色谱硅胶(300 400 目)均为 青岛海洋化工厂生产; BHC-1300 II A/B3 型超净工作台 为苏州净化有限公司生产; YXQ-LS-50S II 立压式力蒸 
汽灭菌器由上海博讯实业有限公司医疗设备厂; HPLC 柱用江苏汉邦 Dubhe TM C18, $10 \mu \mathrm{m}, 20 \mathrm{~mm} \times 250 \mathrm{~mm}$, $\mathrm{YMC} \mathrm{C} 18,5 \mu \mathrm{m}, 10 \mathrm{~mm} \times 250 \mathrm{~mm}$.

\section{2 实验方法}

\subsection{1 科罗索酸 $(\mathrm{CA})$ 的微生物转化篮选}

在无菌条件下, 选择 10 株的细菌和 10 株真菌接于 $100 \mathrm{~mL}$ 的三角瓶内装 $40 \mathrm{~mL}$ PDA 液体培养基中, 放于 TS-2102C 型恒温震荡培养箱进行培养, $2 \mathrm{~d}$ 后加入底物 $\mathrm{CA}$, 共培养 $5 \mathrm{~d}$ 后乙酸乙酯等体积萃取 2 次, 同等条件 下以培养基和不加底物的菌株作为对照, TLC 检测转化 反应.

\subsection{1 科罗索酸 $(\mathrm{CA})$ 的放大培养及转化产物分离}

阳性菌株的放大培养同样采用两步发酵法. $12 \mathrm{~L}$ 液 体培养基中共加入 $600 \mathrm{mg}$ 底物. 发酵液乙酸乙酯提取 减压浓缩后通过硅胶柱色谱(石油醚: 乙酸乙酯), TLC 点板合并相同组分, 组分 Fr.312 经乙酸乙酯重结晶得 13 $\operatorname{mg} 2 \alpha, 3 \beta, 7 \beta$-三羟基-11-烯-13,28-内酯(2), 白色粉末. ${ }^{1} \mathrm{H}$ NMR (DMSO- $d_{6}, 400 \mathrm{MHz}$ ) 6.02 (br d, $J=10.4 \mathrm{~Hz}$, H-12), 5.71 (dd, $J=1.2,9.6 \mathrm{~Hz}, \mathrm{H}-11$ ), 2.88 (d, $J=9.6 \mathrm{~Hz}$, $\mathrm{H}-3$ ), 3.83 (dd, $J=4.8,9.6 \mathrm{~Hz}, \mathrm{H}-7$ ), 3.63 (td, $J=4.8,9.6$ Hz, H-2), 0.97 (s, H-23), 0.92 (d, $J=9.6$ Hz, H-30), 0.92 (s, H-25), 098 (s, H-26), 0.76 (s, H-24), 1.23 (s, H-27), 0.99 (d, H-29); ${ }^{13} \mathrm{C}$ NMR (DMSO- $\left.d_{6}, 100 \mathrm{MHz}\right) \delta: 182.6$ (C6), 133.6 (C12), 130.6 (C11), 91.9 (C13), 84.1 (C3), 73.6 (C7), 69.3 (C2), 61.9 (C18), 53.8 (C9), 53.6 (C5), 48.3 (C-14), 47.4 (C17), 46.5 (C8), 44.1 (C20), 41.5 (C4), 40.3 (C19), 39.2 (C1), 38.4 (C10), 32.5 (C22), 31.7 (C21), 30.4 (C6), 29.2 (C15), 28.8 (C23), 23.9 (C16), 19.4 (C30), 19.2 (C25), 18.3 (C26), 16.9 (C24), 16.8 (C27), 14.9 (C29); HR-ESI-MS cacld for $\mathrm{C}_{30} \mathrm{H}_{47} \mathrm{O}_{5}[\mathrm{M}+\mathrm{H}]^{+}$ 487.3423, found 487.3403 .
辅助材料(Supporting Information) 新化合物 2 的 HRESI-MS, ${ }^{1} \mathrm{H}$ NMR, ${ }^{13} \mathrm{C}$ NMR, HSQC 和 HMBC 谱图. 这 些材料可以免费从本刊网站(http://sioc-journal.cn/)上下 载.

\section{References}

[1] Lin, G.-R.; Shen, G.-Y.; Wu, J.-C. Chin. Agric. Sci. Bull. 2014, 28, 133 (in Chinese).

(林国荣, 沈高扬, 吴锦程, 中国农学通报, 2014, 28, 133.)

[2] He, J.-R.; Liu, J.; Bai, Z.-L.; Huang, R.-Q.; Wang, Z.-L. J. Southern Med. Univ. 2010, 11, 2533 (in Chinese).

(贺建荣, 刘欠, 白志龙, 黄仁权, 王增禄, 南方医科大学学报, 2010, 11, 2533.)

[3] Sung, B.; Kang, Y. J.; Kim, D. H.; Hwang, S. Y.; Lee, Y.; Kim, M.; Yoon, J.-H.; Kim, C. M.; Chung, H. Y.; Kim, N. D. Int. J. Mol. Med. 2014, 33, 943.

[4] Kim, J.-H.; Kim, Y.-H.; Song, G.-Y.; Kim, D.-E.; Jeong, Y.-J.; Liu, K.-H.; Chung, Y.-H.; Oh, S. Food Chem. Toxicol. 2014, 67, 87.

[5] Aguirre, M. C.; Delporte, C.; Backhouse, N.; Erazo, S.; Letelier, M. E.; Cassels, B. K.; Silva, X.; Alegría, S.; Negrete, R. Bioorg. Med. Chem. 2006, 14, 5673.

[6] Xu, Y.-F.; Zhao, Y.-H.; Xu, Y.-L.; Guan, Y.; Zhang, X.; Chen, Y.; Wu, Q.; Zhu, G.-Q.; Chen, Y.-X.; Sun, F.-Y.; Wang, J.-Y.; Yu, Y.-C. Cell Signal 2016, 29, 209.

[7] Ren, X.-H.; Lu, X.-F. Prog. Pharm. Sci. 2011, 3, 129 (in Chinese). (任新凤, 陆雪芬, 药学进展, 2011, 3, 129.)

[8] Park, C.; Lee, J.-S. Biomed. Res. 2013, 24, 164.

[9] Giampapa, V. C.; Falls, L. WO 2006127779, 2006 [Chem. Abstr. 2005, 145, 511747].

[10] Deng, J.-J.; Lu, C.-H.. Nat. Prod. Res. 2015, 30, 1

[11] Feng, X.; Li, D.-P.; Chu, Z.-Y. Nat. Prod. Res. 2014, $28,1$.

[12] Li, D.-P.; Feng, X.; Chu, Z.-Y.; Guo, F.-F.; Zhang, Z.-S. J. Asian Nat. Prod. Res. 2013, 15, 789.

[13] Fu, S.-B.; Yang, J.-S.; Cui, J.-L.; Feng, X.; Sun, D.-A. Chem. Pharm. Bull. 2011, 59, 1180.

[14] Fu, S.-B.; Yang, J.-S.; Cui, J.-L.; Sun, D.-A. Fitoterapia 2013, 86, 123.

[15] Feng, X.; Lu, Y.-H.; Liu, Z.; Li, D.-P.; Zou, Y.-X.; Fang, Y.-Q.; Chu, Z.-Y. J. Asian Nat. Prod. Res. 2016, 30, 1. 